\title{
Un nouvel horizon génétique : la bande dessinée
}

\author{
Pierre-Marc de Biasi et Luc Vigier
}

\section{(2) OpenEdition}

Journals

Édition électronique

URL : http://journals.openedition.org/genesis/1434

DOI : 10.4000/genesis. 1434

ISSN : 2268-1590

\section{Éditeur :}

Presses universitaires de Paris Sorbonne (PUPS), Société internationale de génétique artistique littéraire et scientifique (SIGALES)

\section{Édition imprimée}

Date de publication : 12 décembre 2016

Pagination : 7-16

ISBN : 9791023105490

ISSN : 1167-5101

\section{Référence électronique}

Pierre-Marc de Biasi et Luc Vigier, « Un nouvel horizon génétique : la bande dessinée », Genesis [En ligne], 43 | 2016, mis en ligne le 27 février 2017, consulté le 24 septembre 2020. URL : http:// journals.openedition.org/genesis/1434; DOI : https://doi.org/10.4000/genesis.1434 


\section{Un nouvel horizon génétique : la bande dessinée}

\section{L'émergence d'une épistémè}

Le glissement progressif de la bande dessinée du statut de simple produit de divertissement vers celui de véritable genre artistique s'est traduit depuis une vingtaine d'années par un développement particulièrement fécond d'études théoriques et esthétiques. Le fameux - et laborieux - processus de légitimation, décrit par Luc Boltanski en 1975, a non seulement fondé en raison et valorisé la figure, souvent double ou triple, de «l'auteur» de bande dessinée, mais il a aussi permis l'éclosion d'un nouveau discours critique sur les innovations formelles et les expérimentations graphiques propres au genre, en offrant au milieu universitaire le droit de constituer en objet de recherche ce qui avait spontanément fasciné le public depuis longtemps. Comme pour beaucoup de nouveaux horizons intellectuels, c'est le «moment théorique» des années soixante et soixante-dix qui a redessiné les frontières : en 1976, l'historique numéro 24 de la revue Communications ( La bande dessinée et son discours 1 »), avec les admirables articles de Michel Covin ( "L'image dérobée »), d'Umberto Eco ( LL mythe de Superman») ou de Pierre Fresnault-Deruelle ( $D$ Du linéaire au tabulaire»), marque en France l'entrée officielle de la BD dans le champ des sciences de l'homme et des études sémiologiques. Mais la curiosité des lecteurs en la matière est en réalité beaucoup plus ancienne. Même dans leurs manifestations les plus simples, sous forme de strips ou de feuilletons, ces performances visuelles n'ont en fait jamais cessé de fasciner, largement au-delà du jeune public et clairement depuis les tout premiers moments de la bande dessinée, lesquels remontent bien avant les grands succès commerciaux franco-belges des années trente ou les comics américains, et se situent vers la fin du XIXe siècle, au moment où les rotatives de la presse imprimée commencent à tourner à plein régime, dans la période qui voit naître aussi le cinéma et l'animation.

C'est en observant le travail d'artistes comme Hogarth, McCay, Töpffer et Rabier, au contact de la peinture, des beaux-arts en général, de la publicité, des formats de presse et un peu plus tard des dessins animés, que s'est formée l'idée selon laquelle, toute populaire qu'elle puisse paraître, la bande dessinée est un mode d'expression qui, depuis ses débuts, relève du littéraire et de l'artistique, mais qui se caractérise aussi par une ouverture spontanée aux médias contemporains : un médium doué de synergie puisqu'il manifeste une capacité sans précédent à jouer sur toutes les interactions possibles avec l'image, le texte, le scénario, l'intrigue, le gag, la peinture, l'architecture, le théâtre et les arts de l'image animée; mais également un médium opportuniste, puisqu'il se montre capable de muter à vue comme un virus, et de s'adapter en s'appropriant les nouveaux dispositifs d'écriture, de lecture et de réalisation graphique au fur et à mesure de leur émergence technique, de leurs perfectionnements et de leur évolution conceptuelle.

1. À lire en ligne : < www.persee.fr/issue/comm_0588-8018_1976_num_24_1> 
Ainsi en est-il de la bande dessinée contemporaine et spécialement de la bande dessinée qu'on pourrait nommer d'avant-garde, qui intéresse la recherche, au point que certains dessinateurs, par inclination naturelle ou par nécessité, sont parfois devenus eux-mêmes théoriciens de leur propre pratique ou même historiens du genre, passant allègrement d'un côté à l'autre du miroir pour explorer ce qu'il faut bien reconnaître désormais comme une histoire mondiale de la bande dessinée : une histoire où les mouvements, les tendances, les ruptures, les remises en cause, les modes, les styles, les maîtres, les petits maîtres, les imitateurs, les découvreurs, etc. se succèdent comme dans toutes les autres formes d'art. Et c'est ainsi que du dessin de presse de la Belle Époque aux webcomics des supports numériques, la bande dessinée se métamorphose et se développe depuis plus d'un siècle en se renouvelant à la faveur de ses propres crises et de ses propres révolutions. Cette histoire se caractérise, y compris à travers les auvres en apparence les plus normées, comme la recherche permanente de formes inédites, comme une sorte de besoin permanent de renaissance et de redéfinition où semble se jouer un dynamisme créatif n'ayant rien à envier aux révolutions qui ont traversé les autres univers artistiques du siècle.

Propulsée dans les années soixante-dix par des magazines qui tenaient le milieu entre les supports de création les plus novateurs et les blockbusters graphiques les plus orientés «industrie culturelle de masse », la production dite "commerciale » a explosé dans les années quatre-vingt-dix dans toutes les directions, au point que les libraires spécialisés qui veulent rendre compte d'une part significative de la création contemporaine sont aujourd'hui soumis à un turn over aussi épuisant en termes de quantité qu'en termes de qualité. L'énormité de la production les contraint à un arbitrage esthétique, intellectuel et plastique particulièrement exigeant : le libraire doit opérer une sélection permettant de mettre en lumière les meilleures «nouveautés», mais il ne peut pas non plus négliger complètement le reste des parutions, si bien qu'il lui est finalement impossible d'accorder aux ouvres les plus importantes mieux qu'une mise en valeur très courte, de quelques semaines. Parallèlement à cette production commerciale, on a vu émerger - à partir de Maus d'Art Spiegelman au milieu des années quatre-vingt-des œuvres de nature profondément différente, affichant un nombre de pages inaccoutumé, une ambiance et un traitement des figures inédits, se détachant parfois de la couleur pour retrouver le dessin linéaire pur, développant un dispositif de cases simplifié et spontané : cette mouvance, qui rejoignait à l'époque un courant littéraire focalisé sur l'intime, l'autobiographique, l'autofiction et le sociologique, a donné naissance à ce que l'on a appelé, faute de mieux, le roman graphique.

C'est également dans les années soixante-dix - non sans lien, souvent souligné par les dessinateurs de la période, avec la déferlante musicale rock des grands groupes anglais et américains - que quelques communautés fondent par dizaines des fanzines underground, tandis qu'au même moment, dans le prolongement de cet enthousiasme, d'autres passionnés expérimentent les possibilités infinies des algorithmes visuels ou la torsion du temps et de l'espace (Oubapo). Que ces initiatives aient réactivé l'idée d'une littérature graphique (Töpffer parlait de "Littérature en estampe» et Thierry Groensteen évoque ici la bande dessinée comme "une littérature») ne peut surprendre, tant les liens que la bande dessinée manifeste avec les arts de l'écrit - et particulièrement avec le roman - sont aussi anciens et prégnants que ceux qu'elle entretient avec les arts visuels, la musique, la publicité et le design. Plus subtilement, la relation entre roman et bande dessinée semble s'être cristallisée 
autour d'une homologie : leur liberté et leur égale impunité formelle à l'égard des règles. Le roman, comme la bande dessinée, est l'expression et le lieu même d'un genre sans foi ni loi, habile dans l'art de parler de tout et de rien, apte à tout aborder et à tout absorber, adroit dans l'imitation de la réalité mais tout aussi crédible dans ses plus irréelles fantasmagories, expert en contradictions, capable de s'adapter et même de se métamorphoser, de se renverser du pour au contre, jusqu'à enfreindre toutes les conditions de sa légitimité sans risquer de perdre sa qualification générique.

\section{Invitations}

La singularité de la bande dessinée dans la construction du récit, des figures et des voix, dans l'exploration des sensations et de la pensée, la synesthésie graphique dont elle est capable, sa puissance sémiotique, sa capacité presque naturelle à inventer des mondes, son sens aigu de la fiction, sa recherche de dispositifs, la spécificité de ses techniques croisées - qui font de la bande dessinée à jamais un art du mixte, du mélange, de l'alchimie, de l'hybride - font de ses productions icono-textuelles à la fois l'expression d'une diversité à investir et l'incarnation exemplaire d'un processus créatif qui intéresse naturellement la génétique des auvres et de la création. Réciproquement, les approches de la génétique, en ouvrant les dossiers de dispositifs d'écriture et de lecture encore mal connus, en offrant la possibilité de saisir les mécanismes d'engendrement de l'image, peuvent créer des entrées nouvelles dans un domaine bien moins fréquenté que les brouillons de Proust et de Flaubert. La médiatisation de la bande dessinée a certes donné à voir quelques événements de coulisses, dès les années soixante-dix, à l'instar des Cahiers de la bande dessinée où la Une était régulièrement occupée par une représentation parodique mais très précise de l'artiste en son atelier. Même si très souvent, par effet d'époque, le ton était volontiers auto-ironique, on percevait très clairement, à travers les fragments de documents de travail reproduits et à travers les interviews de dessinateurs et scénaristes, non seulement la superposition des tracés, des encres et des couleurs, mais aussi la multiplicité des travaux préparatoires, des essais, des études - y compris chez des dessinateurs dont le trait pouvait passer pour simple à l'extrême.

Quelques documentaires de télévision s'intéressant à la virtuosité ou à la technique du tracé firent alors leur apparition : le médium dominant filmant le médium marginal, ils présentaient le dessin comme spectacle, valorisaient l'improvisation en direct, le cadavre exquis, le miracle de la main. Plus récemment, des chaînes culturelles ont un peu approfondi le sujet en consacrant quelques courts-métrages à des humoristes et à des dessinateurs de presse, quelques-uns portant leur attention sur les créations collaboratives pour tenter de saisir le mystère de cette création à deux têtes (évoqué par Benoît Peeters dans l'entretien qu'il nous a accordé). Plus récemment encore, alors que se développe, par ailleurs, le goût des making of d'albums, selon une technique empruntée au cinéma d'animation, c'est sur les chaînes du réseau Internet que d'innombrables films de dessinateurs au travail, souvent tournés en accéléré, sont devenus un véritable genre, un art de la performance, renvoyant le dessin de bande dessinée à la magie, à l'hypnose, à l'incroyable vertige de la virtuosité technique (voir la contribution de Luc Vigier). Parallèlement à ces manifestations filmiques qui cherchent à reconstituer une image de la genèse, l'intérêt visible des éditeurs pour les 
carnets de création (études, esquisses, brouillons), les cahiers de travaux préparatoires qui se trouvent ajoutés aux albums, sont autant de signes d'une passion grandissante du public pour les secrets de fabrication, pour l'euvre à l'état naissant, pour l'atelier de la BD. Les quatre cents planches originales, esquisses et crayonnés présentés par la toute récente exposition Hergé au Grand Palais à Paris (septembre 2016-janvier 2017) confirment on ne peut plus clairement le phénomène. Un tel mouvement corrobore les arguments des chercheurs qui plaident pour une exploitation plus systématique des données antérieures à l'ouvre définitive.

\section{Assimilation}

Ce numéro de Genesis constitue à ce titre une première et une ouverture nouvelle : il fait entrer les processus mentaux, mémoriels, scripturaux, iconographiques et techniques de la bande dessinée dans le spectre analytique et épistémique de la génétique des arts. Les enjeux d'une génétique de la bande dessinée doivent leur tressage à la forte densité des processus endogénétiques, ramifiés par l'intervention d'instances multiples (auteurs, dessinateurs, scénaristes, coloristes, éditeurs, traducteurs) et la multiplication des processus d'influence exogénétiques appelés naturellement par un art d'une grande porosité esthétique, culturelle et plastique. De fait, les dossiers génétiques atteignent des volumes importants où se croisent les planches originales, les différentes étapes de leur achèvement ou de leur perfectionnement avant édition. Plans, scénarios, storyboards de toutes formes, dessins de scénaristes, spectres graphiques d'une intention, études, esquisses, carnets de croquis, projets abandonnés, recyclés ou augmentés, textes, photographies, coupures de presse, maquettes, ou même objets, comme dans la récente exposition des planches originales de Jean-Christophe Menu où l'on pouvait apercevoir, sous vitrine, les objets fétiches fréquemment représentés dans ses albums.

Ces traces multiples et diversifiées de l'itinéraire de création sont autant d'indices qui aident à comprendre la complexité du cheminement par lequel le créateur se donne un maximum de moyens pour inventer en toute liberté sans cesser de respecter les contraintes plus ou moins indépassables de la planche : le cadre d'expression qui est à la fois une attente du lectorat, de l'éditeur, et la base référentielle du dispositif technique qui permet de produire l'album. Les exigences du récit, l'invention du milieu narratif, la lente construction des personnages, des décors et des mondes, l'émergence d'idées nouvelles, voilà ce qui se donne à lire et à interpréter dans ces dossiers foisonnants, au gré des nombreuses variations et métamorphoses souvent développées sur plusieurs années. Mais à travers cette multitude de traces de l'invention endogénétique, ce qui se présente à nous, c'est aussi tout ce dont la bande dessinée se saisit et s'empare pour fabriquer la matière exogénétique qu'elle manipule, s'approprie et recycle : ce qu'elle emprunte aux beaux-arts, au plan technique aussi bien qu'esthétique, tout ce qu'elle accapare, assimile et modifie ou subvertit des arts de la photographie, de la peinture, du film, de l'animation, sans oublier, bien sûr, le domaine où elle exerce ses talents de prédateur les plus accomplis et les plus impitoyables: le monde de la BD elle-même, son actualité, son histoire et sa culture.

Sur tous ces phénomènes de capture et de conversion qui placent la création exogénétique au cour des processus d'émergence du nouveau, l'approche génétique est la mieux armée - probablement même la seule plausible - pour reconstituer les logiques secrètes par 
lesquelles l'œuvre originale et le style personnel de l'auteur s'élaborent en prélevant leurs matériaux et leurs ressources formelles dans l'extériorité du déjà-créé. Mais, précisément, pour mesurer et comprendre comment de l'extériorité devient de l'intériorité, encore faut-il pouvoir disposer de dossiers de genèse suffisamment complets. Comme le prouvent leurs archives et leurs expositions, les créateurs de $B D$, attentifs à leur processus de création et à la préservation de leurs ressources documentaires, sont souvent d'excellents conservateurs de leurs propres dossiers de genèse (voir les contributions de François Matton et Alexandre Clérisse), parfois jusqu'à la précision exhaustive de la démarche obsessionnelle, du classement monomaniaque et du fétichisme. C'est un travers qu'il faut chaudement encourager, car c'est une chance inespérée pour l'avenir de toutes les recherches en génétique de la BD. La littérature nous a montré qu'aucun dossier de genèse ne se conserve efficacement s'il ne bénéficie pas de sollicitudes préalables tout au long de la vie de son auteur. Si ces conditions sont réunies, la technologie permet aujourd'hui d'envisager la suite avec optimisme: les possibilités de conservation et d'étude fournies par l'imagerie et la numérisation offrent désormais à la BD les moyens d'une capitalisation massive des documents de genèse et par conséquent la promesse d'une analyse approfondie des sentiers de la création.

\section{Bruissement réflexif des images}

À plus d'un titre, la bande dessinée est profondément, viscéralement, structurellement hypertextuelle, hypericonique et hypercitationnelle. Il y a un bruissement iconique comme il y a pour Barthes un bruissement de la langue. La bande dessinée fait constamment allusion à. Elle cite, reprend, parodie, copie, contrefait, dissimule, exhibe, manifeste à tout instant ses héritages, joue et se joue de tous les niveaux d'interaction entre les images présentes et les images fantômes. Très souvent aussi, elle pratique le collage, par reprise et dépassement d'images antérieures, dans des processus qui, ici comme ailleurs, peuvent relever, selon les cas, du travail iconoclaste ancien, moderne ou postmoderne. Les parodies de Tintin, ou encore les effacements graphiques de Jochen Gerner dans TNT en Amérique ${ }^{2}$ (gommant tout des cases de Tintin en Amérique et réduisant les planches à des suites de signes abstraits) sont typiques d'une présence endémique de la réflexivité au cœur du régime de signification propre à la bande dessinée. Se regardant fonctionner, accoutumée à produire, plus ou moins discrètement, une forme de métadiscours théorique, la bande dessinée porte une attention professionnelle, constante, productive et souriante sur sa propre genèse.

Et voilà pourquoi, d'une manière un peu exceptionnelle dans la revue Genesis, nous avons décidé, pour ce numéro, de donner résolument la parole aux auteurs eux-mêmes-scénaristes et dessinateurs - en leur demandant de se faire les observateurs de leurs propres démarches et les analystes de leurs propres processus de création, tout comme nous l'avions fait, naguère (Genesis, $n^{\circ} 14,2000$ ), pour une autre discipline qui pratique professionnellement l'autoanalyse génétique : l'architecture. Si la bande dessinée est autoréflexive, c'est, comme dans le processus de conception architecturale, parce que chaque décision de détail engage, de proche en proche, l'ensemble du projet et que le créateur ne peut avancer vers un résultat final qu'en se ménageant en permanence la possibilité de revenir en arrière dans son travail,

2. Jochen Gerner, TNT en Amérique, Paris, L'Ampoule, 2002. 
en remontant dans l'arborescence de ses choix pour sortir d'une impasse ou trouver une meilleure solution. Créer suppose donc à chaque moment une vision claire des étapes qui précèdent, mais cette attention que la bande dessinée porte à sa propre production n'est pas non plus dénuée d'humour ni de mise en abyme.

De l'ironie savoureuse d'un Gotlib aux expérimentations de Chris Ware ou de Trondheim, elle est devenue un art spéculatif et spéculaire qui échafaude ses fictions en déconstruisant à vue ses propres processus. C'est dans cette logique, et en écho, que sont récemment apparus, simultanément dans le monde de la bande dessinée et dans celui de la recherche, des essais graphiques monumentaux: des thèses en bandes dessinées sur la bande dessinée (Unflattening 3 , de Nick Sousanis, par exemple, et plus anciens, les ouvrages de Scott Mac Cloud sur les techniques du récit), de véritables recherches rédigées en images pour penser la fabrique mentale des personnages, de la fiction, du temps, de l'espace, des scénarios.

Ces phénomènes confirment, s'il était nécessaire, que le dispositif de la bande dessinée se constitue spontanément en discours sur lui-même et qu'une génétique de la bande dessinée ne constituerait au fond qu'une réponse naturelle aux multiples sollicitations et appels que nous lancent depuis longtemps une masse considérable de dossiers autographes et une multitude de chantiers graphiques, parfois exposés comme à ciel ouvert, qui se présentent à notre attention dans leur intégralité génétique et esthétiquement à part entière comme des ouvres à l'état naissant. Une génétique de la bande dessinée commence donc à prendre au sérieux ce mouvement d'autoanalyse et d'auto-épistémologie. Elle vient globalement accompagner l'articulation de la bande dessinée avec tout ce qu'elle capte, absorbe, transforme, au cours d'une alchimie exogénétique à large spectre où elle se crée en cherchant le contact avec des éléments idéologiques, historiques, esthétiques qui la transforment de l'intérieur en une sorte de précipité sociohistorique : le révélateur d'une autorité spéciale pour parler de l'état présent du monde, de ses espoirs, de ses cauchemars, de son avenir. C'est le cas, bien sûr, pour toute ouvre d'art digne de ce nom, mais la BD a ceci de particulier que cette auctoritas s'élabore à l'échelle des ressources scriptographiques les plus modestes, dans le travail de pure recherche des formes, dans ce que les tracés font apparaître comme intention de sens, dans l'énergie des mises en couleurs, bref dans les données élémentaires de l'image où se jouent les conversions massives de l'exo-en endogenèse (voir l'inédit de Calvo par Jean-Pierre Mercier ou les travaux de Gérald Gorridge).

\section{Séquences de séquences}

Mais l'approche génétique de la BD ne peut s'en tenir à l'analyse du dessin ou de la planche en tant que tels: ces unités ne s'entendent que rapportées à la temporalité globale d'un programme, qui en font les unités d'une histoire séquentielle à plusieurs degrés : celle de l'œuvre. S'agissant d'une ouvre conçue comme un livre et destinée à être "lue » page à page, on se trouve face à la question fondamentale des séquences successives de travail, dans une relation d'analyse qui peut se comparer à celle que l'on a déjà théorisée pour les dossiers génétiques d'écrivains, mais avec, de surcroît, la force de frappe de l'image à la

3. Nick Sousanis, Unflattening, Cambridge, Harvard University Press, 2015; Le Déploiement, version française parue chez Actes Sud/Éditions de l'An 2 (traduction de Marc Voline et préface de Thierry Smolderen), 2016. 
naissance et aux métamorphoses de laquelle, par le miracle des esquisses et des documents de genèse, il devient possible d'assister. Au-delà du narratif et du modèle littéraire, la séquentialité du processus de fabrication des séquences d'images fait donc apparaître une dimension graphique, qu'une approche des archives de la bande dessinée doit pouvoir décrire, en grande partie (comme le montre ici Pierre-Marc de Biasi) à partir des outils et descripteurs forgés à la faveur des études génétiques déjà réalisées sur des corpus graphiques, plastiques et iconiques. Combinée aux ressources de la génétique littéraire, cette approche est aujourd'hui en état de révolutionner l'étude d'un des domaines les plus vivants de la création. C'est de ce cadre méthodologique et scientifique déjà en état de fonctionner qu'il s'agit désormais de penser l'institutionnalisation en termes d'outil de recherche collective. Les fonds d'archives sont disponibles. Il ne reste plus qu'à les rendre scientifiquement opérationnels en réalisant leur jonction avec les organismes de recherche qui ont prouvé leur excellence en matière d'analyse génétique.

Plusieurs lieux, en France, en Belgique, en Angleterre, aux États-Unis, par exemple, ont eu le réflexe heureux, au début des années quatre-vingt, d'acquérir les brouillons et de conserver les collections, engageant parfois de coûteux travaux de restauration. C'est le cas du Musée de la bande dessinée à Angoulême, où les collections sont protégées exactement comme cela se ferait dans les départements spécialisés de la Bibliothèque nationale. Mais cela peut aussi prendre la forme de dons massifs, comme François Schuiten l'a voulu, en 2013, léguant l'ensemble de ses dossiers génétiques et planches originales à plusieurs institutions belges, répliquant aussi le geste hugolien du grand legs de la fin du XIXe siècle, par lequel Victor Hugo fondait en France, par son codicille de 1881, la possibilité d'une étude généticienne de ses auvres, et notons-le, de la totalité de ses ceuvres aussi bien littéraires que plastiques : "Je donne tout ce qui serait trouvé écrit ou dessiné par moi à la Bibliothèque nationale... » L'exposition, de plus en plus fréquente, de planches originales, qui se fait dans les musées graphiques dans un état d'esprit bien différent des salles d'enchères de Sotheby's ou Drouot, se place de fait dans une démarche pédagogique et scientifique anticipant clairement les enjeux d'une génétique active de la bande dessinée qui pourrait prendre la forme de laboratoires d'études, en lien direct avec les lieux de conservation.

Si l'on compare la situation à ce qui pouvait être dit dans cette revue, il y a quinze ans, des archives architecturales ${ }^{4}$ ou, en 2003 , des archives scientifiques ${ }^{5}$, on constate une évolution remarquable. En 2000, le numéro de Genesis consacré à l' «Architecture » s'ouvrait sur un débat d'idées concernant les urgences en matière de conservation patrimoniale, et notamment sur l'alternative de la «collection» ou du «fonds » : beaucoup d'institutions à l'époque (quelques-unes aujourd'hui encore, avouons-le) préféraient consacrer leurs dotations annuelles ou exceptionnelles à l'acquisition très onéreuse d'un ou deux dessins spectaculaires de très grands architectes (faciles à exposer et à valoriser auprès du grand public) plutôt qu'à l'achat de fonds entiers d'agences, constitués d'esquisses et de graphismes moins prestigieux mais souvent bien plus riches du point de vue de la recherche. On observait déjà à l'époque une vraie réflexion à ce sujet et une tendance à se réorienter vers l'hypothèse

4. Genesis, n 14, «Architecture», dir. Pierre-Marc de Biasi et Réjean Legault, 2000.

5. Genesis, $n^{\circ}$ 20, «Écriture scientifique», dir. Anouk Barberousse et Laurent Pinon, 2003 (sur la question des fonds, voir notamment P.-M. de Biasi «Sciences, des archives à la genèse», p. 19-52). 
scientifique. On constate que ces dernières années, la BD, dans bien des cas, a jeté les bases d'une politique archivistique responsable, en optant résolument pour la bonne formule : la constitution de fonds, au sens génétique du terme.

Il reste évidemment beaucoup à faire, mais ce qui est acquis est déjà un immense gage d'avenir. Les dossiers disponibles sont parfois très complets, parfois lacunaires : si les planches originales et les crayonnés ou les esquisses sont aujourd'hui conservés par leurs auteurs avec beaucoup d'attention, notamment en raison des prix élevés qu'ils peuvent atteindre sur le marché, et si, de même, les carnets font partie des outils de travail auxquels les dessinateurs tiennent particulièrement (voir ici les contributions de Sabine Teyssonneyre, de Gérald Gorridge et de Johanna Schipper), il n'en est pas de même des documents textuels, des correspondances papier ou numériques, des notes ou des dossiers documentaires que les auteurs n'ont pas toujours songé à conserver, ou que les contributeurs d'une même œuvre peuvent avoir gardés séparément.

Ce numéro rebrasse à cet égard certains des enjeux soulevés par le numéro 41 de Genesis sur le travail collaboratif 6 : les dossiers peuvent en effet cumuler des documents issus de plusieurs auteurs à la fois qui échangent d'autant plus volontiers, aujourd'hui à distance, que le numérique leur fournit des possibilités quasi immédiates de transactions, de dialogues et d'arbitrages au moment du travail créatif (mails, envois de versions, de projets de planches, de couleurs, de textures, échanges avec l'équipe de fabrication, corrections, interventions, négociations diverses), mais leur offre aussi un moyen d'échange permanent pour élaborer leurs projets au fil des années de collaboration. Ces correspondances numériques suivies, avec leurs interruptions, leurs périodes de haute intensité, leurs documents annexés, etc. constituent des données évolutives de premier plan pour comprendre l'amont des créations sur une grande échelle spatiale et un large empan chronologique. Ces archives ne sont pas moins riches d'enseignement pour comprendre l'aval des æuvres et le rôle d'intervenants extérieurs : pensons, par exemple, aux interventions parfois déterminantes de l'éditeur, à la naissance du projet, puis pendant le processus et enfin après lui, lorsqu'il s'agit de comprendre dans la genèse le rôle souvent crucial des contraintes de formes, de temps, de financement qui ont pu peser sur les choix, ou au contraire offrir de nouvelles perspectives. Ce type d'archives est également décisif lorsqu'il s'agit de reconstituer la phase éditoriale, et spécialement, en aval de l'édition, les problèmes posés par la traduction de l'œuvre dans d'autres langues. En littérature, la génétique, plus que toute autre méthode d'analyse des textes, est attentive à la part souvent considérable de création qui anime le processus même de la traduction de l'œuvre. Mais lorsqu'il s'agit de penser la traduction de la bande dessinée, la question se pose avec une acuité encore plus vive: qu'est-ce que traduire une image, comment s'articulent les liens entre image et texte dans la traduction d'une BD? À une époque où le genre bénéficie d'un marché éditorial qui se mondialise, des questions nouvelles émergent à cet égard: comment traduire une ouvre de BD occidentale, et la rendre interprétable au sens plein du terme, pour des cultures extrêmes-orientales comme celles - si riches en ce domaine - du Japon ou de la Chine? Et réciproquement... À quelles transpositions du dispositif visuel lui-même faut-il recourir, dans l'un et l'autre sens, pour opérer une traduction digne de ce nom? Ne s'agit-il pas alors, pour une part, d'une nouvelle genèse?

6. Genesis, n 41, «Créer à plusieurs mains », dir. Nicolas Donin et Daniel Ferrer, 2015. 


\section{Un texte pour l'image}

La parenté des processus de la bande dessinée avec la génétique des textes ne doit pas masquer que les données scénaristiques, par exemple, mais aussi les recherches, les listes, les concepts, les éléments de dialogue, les descriptions de décors, de situations sont, comme au cinéma, des textes orientés-image qui ne sont donc pas destinés à valoir pour eux-mêmes ni même à se maintenir en tant que tels dans l'œuvre. Le plus souvent, ils aspirent à leur complète disparition. Les textes préparatoires, quand ils existent (puisque dans certains cas l'image précède le texte) ne sont tout au plus que la source, le code, le sous-langage de l'image qui en sera la traduction graphique, avec toutes les mutations et toutes les métamorphoses que cette transposition implique. Le texte scénaristique contient aussi-même dans le cas des bandes dessinées muettes - le discours, les dialogues et les récitatifs. Mais, comme le souligne Thierry Smolderen, c'est dans cet intervalle entre le texte chargé d'intentions iconiques (qui en fait un texte suspendu, un texte-pour-l'image, un texte en attente d'effacement partiel) et sa nécessaire disparition que l'essentiel d'une recherche génétique se joue. Il y a un amont verbal de la composition de l'intrigue, mais la narrativité de l'auvre comme l'ensemble de sa cohérence artistique et sémiotique ne se gagnent qu'au prix d'une disparition massive du textuel au profit du visuel : interpréter la genèse c'est comprendre par quels processus ceci tue cela.

$C$ 'est ce qui distingue radicalement une génétique des textes et une génétique de la BD. On pourrait en rester à ce constat. Mais il y a aussi ce qui distingue une génétique de la $B D$ et une génétique des arts purement visuels, de la peinture par exemple. À la différence du tableau, l'image y fût-elle dominante, une œuvre de BD achevée se "lit», et quand on dit "lire», précisément, il ne s'agit pas d'une image : la BD n'est pas synoptique, elle reste assujettie à la séquentialisation du texte, même si le texte qui l'a produit a fini par disparaître. Mais s'agit-il d'un effacement ou d'un recouvrement? d'un gommage ou d'un palimpseste? L'enjeu de la recherche génétique ne consiste donc peut-être pas seulement à comprendre pourquoi et comment ceci (l'image) a tué cela (le texte) mais à se demander de quelle manière le texte mort survit dans l'image. Par un remarquable paradoxe dont l'énigme est sans doute le cour même de l'interrogation génétique, cette disparition qui fait l'œuvre finale n'est parfaitement réussie que lorsqu'elle contient aussi, mystérieusement, le principe d'une rémanence, la vigueur d'un retour actif du refoulé.

Tout se passe comme si, dans l'œuvre finale, la mémoire d'une partition textuelle désormais invisible hantait l'image, lui assignait un sens crypté qu'il s'agit pour le lecteur de reconstituer par les seules ressources du récit visuel offert à sa perspicacité et à ses affects. À la manière du songe, la contiguïté des images, en bande dessinée, nous est donnée à «lire » comme une concaténation de visions traversées par autant d'ellipses. Elle nous est donnée à déchiffrer, case à case, planche après planche, comme un récit dont le contenu manifeste (ce que semble représenter l'image, ce que disent explicitement les bulles et les récitatifs) nous renverrait à la logique d'un autre contenu, latent : un composé mouvant de signifiants verbaux et visuels qui se succèdent selon un rythme à la fois étrange et familier, qui dirait à la fois la syntagmatique de l'image et l'iconicité archä̈que de la parole, une sorte de code perdu venu du fond des âges (de ce lointain passé où nous ne savions pas encore lire?), quelque chose comme une partition qui aurait l'aspect d'un hiéroglyphe. 
Ce second discours, peut-être informulable, qui reste à déchiffrer et à ressentir, l'œuvre ne l'a construit plus ou moins délibérément dans son altérité que pour s'en décharger comme de ce qu'elle nous délègue : comme ce qu'elle remet entre nos mains de lecteur pour faire de nous des interprètes, au sens musical du terme, des créateurs. Les dossiers liés à la fabrique de la bande dessinée nous confrontent ainsi, sans doute pour la première fois, à une forme de cascading génétique - processus génétiques enchaînés - où les médiations et les renversements entre le scriptique et le graphique, entre le verbal et l'iconique, semblent répondre à une injonction aussi puissante que celle de la figurabilité décrite par Freud pour interpréter le travail du rêve. Cette singularité rêveuse de la bande dessinée est probablement structurale : elle exige toute l'attention de ceux qui, à la suite de ce numéro, emboîteront le pas d'une étude inédite du neuvième art, désormais concevable - in statu nascendi - comme un espace de découvertes exemplaires pour les études de genèse.

Pierre-Marc de Biasi et Luc Vigier 